\title{
Benign Early Repolarization Pattern: Is it Really Benign for Children?
}

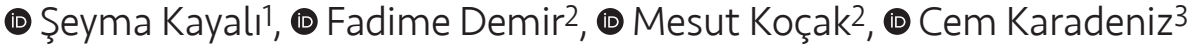 \\ 1University of Health Sciences, Keçiören Training and Research Hospital, Clinic of Pediatric Cardiology, Ankara, Turkey \\ 2University of Health Sciences, Keçiören Training and Research Hospital, Clinic of Pediatrics, Ankara, Turkey \\ 3̇zmir Katip Çelebi University, Department of Pediatric Cardiology, İzmir, Turkey
}

\begin{abstract}
Aim: Early repolarization (ER) is a common electrocardiographic (ECG) finding. Although it is thought to be a benign phenomenon, recent studies have shown it to be an important marker of cardiac vulnerability, which can lead to sudden cardiac death. However, there are still conflicting data regarding the prognostic significance of ER in asymptomatic subjects, especially in children. The aim of this study was to investigate specific ECG markers that reflect ventricular repolarization in children with benign ER.
\end{abstract}

Materials and Methods: The study group included 56 healthy children with a benign ER pattern on ECG and a control group was formed of 81 children with normal ECG. Benign ER pattern was defined as terminal QRS notching or slurring accompanied by rapidly ascending ST elevation (>0.1 mV from baseline) in two or more leads. The ECG parameters of QT dis, QTc dis, Tpe dis, Tp-e/QT and Tp-e/QTc were evaluated at rest by a single experienced pediatric cardiologist blinded to the groups.

Results: Higher Tp-e, Tp-e dis, Tp-e/QT and Tp-e/QTc measurements were determined in the study group than in the control group. There were no significant differences in the studied ECG parameters with respect to ER location (inferior, lateral, inferolateral), or the ER type (slurring, notching or both).

Conclusion: These findings suggest that benign ER in children is associated with the risk of arrhythmogenesis through alterations in ventricular repolarization.

Keywords: Arrhythmia, children, sudden cardiac death

\section{Introduction}

Early repolarization (ER) is universally defined as 0.1 $\mathrm{mV}$ ST segment elevation in at least two adjoining leads (1). In the absence of symptoms, it has been considered as a normal state found mainly in teenagers, youngsters, and athletes $(2,3)$. However, case reports, experimental and epidemiological studies with large samples have shown that ER could be related with arrhythmias leading sudden cardiac death (SCD) $(4,5)$.
Therefore, recent studies have explained this phenomenon by classifying it into benign and malign patterns and have implied that clinical findings and electrocardiographic data are important for differential diagnosis. According to these studies, a benign ER pattern can be briefly defined as I wave distribution mainly in V3-V5 (precordial leads) and II, III, aVF (inferior leads) and rapidly ascending segment of ST (not descending or horizontal) with tall $R$ waves in the absence of symptoms $(4,6-8)$. 
However, this definition is still not consistent in adult studies, and is not known to be valid for the pediatric age group, where this ER pattern is ten times more prevalent than in adults (3).

Furthermore, although there are studies documenting ER patterns on the electrocardiograms (ECG) of healthy children with idiopathic ventricular fibrillation and SCD, data is still scarce about the clinical importance of benign ER patterns in children (3).

In this study, we wanted to examine if there is a risk of arrhythmogenesis in healthy children with a benign ER pattern. ECG markers; including T peak to end interval (Tpe), QT interval (QT), corrected QT interval (QTC), dispersion of these intervals (Tp-e dis, QT dis, QTc dis), Tp-e/QT and Tp-e/QTc were calculated, as these are known to be non-invasive determiners of transmural dispersion of repolarization (TDR), which has been suggested to underlie arrhythmogenesis $(9,10)$.

\section{Materials and Methods}

A retrospective analysis was made of pediatric subjects who presented at the pediatric cardiology department for miscellaneous reasons (chest pain, sports participation, murmur, etc.) and underwent a history and physical examination, baseline electrocardiogram and transthoracic echocardiogram.

History: The children recruited into the study were those with no history of arrhythmia and syncope, no known arrhythmia susceptibility syndrome, no known heart disease, and no family history of SCD. The subjects were asked about participation in regular sports activities and only those with no sports activities or less than one year of regular sports participation were included the study because of the wellknown effect of sports on cardiac structure and ER pattern visualization (11).

Physical examination: Subjects with a good clinical condition with no significant findings of acquired or congenital heart disease, or any other infectious or chronic illness were eligible to participate.

Electrocardiography: An ECG recorder (Nihon Kohden, Tokyo, Japan) set at $25 \mathrm{~mm} / \mathrm{s}$ paper speed and $10 \mathrm{~mm} /$ $\mathrm{mV}$ voltage was used to obtain 12 lead ECG recordings following the history and physical examination. Subjects were excluded from the study if the ECG demonstrated any pre-excitation syndrome, prolonged QTc, arrhythmogenic right ventricular cardiomyopathy or Brugada pattern.

Echocardiography: A Vivid 3 (General Electric, USA) echocardiography device with $3 \mathrm{MHz}$ probes was used to perform echocardiography by a single pediatric cardiologist blinded to the ECG findings of the participants. Only those subjects without pathological valve insufficiency or structural heart disease, with normal systolic function and normal wall diameters according to age were included in the study.

Benign ER determination and analysis of ECG parameters: After primary analysis of the subjects as described above, the ECGs were then reviewed by a physician to avoid interobserver variability, with particular attention to benign ER pattern. The definitions by Tikkanen and Huikuri (6) and Perez-Riera et al. (7) were used to determine subjects with benign ER (12);

1- At least $1 \mathrm{~mm}(0.1 \mathrm{mV})$ but not more than $2 \mathrm{~mm}(0.2$ $\mathrm{mV}$ ) elevation of the J-point (the junction between the QRS complex terminal and the beginning of ST segment) in at least two consecutive leads.

2- J-point elevation (rapidly ascending-not descending or horizontal) manifestation could be QRS slurring (slow deflection of downslope $\mathrm{R}$ wave) or notching (a positive wave at the J-point)

3- These changes can be in II, III, aVF (inferior leads), I, aVL, and V4-V6 (antero-lateral leads) or both. In accordance with previous studies, the V1 to V3 leads were not interpreted to avoid confusion with the phenotype of Brugada syndrome $(5,11)$.

The subjects were subdivided into two groups as those with benign ER pattern and those with no ER pattern. The QT interval was defined as being from the beginning of the QRS complex to the end of the T wave and Tp-e was defined as being from the peak of the $T$ wave to the point at which the $T$ wave returns to the baseline (Figure 1). At least three T waves, QT intervals were evaluated in each derivation and the mean was calculated as milliseconds (ms). QTc interval was calculated according to the Bazett formula.

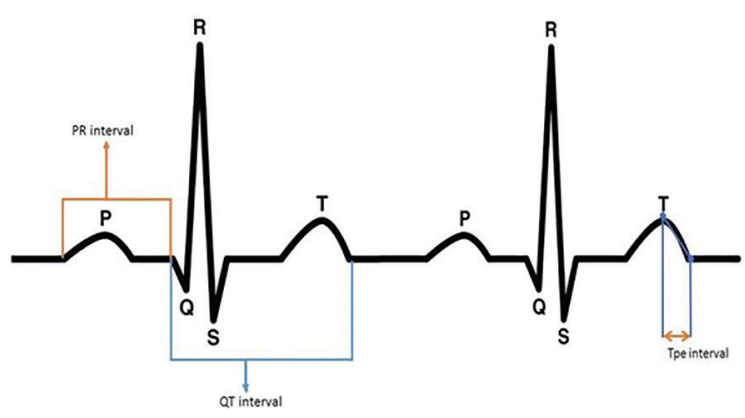

Figure 1. Parameters of ECG

ECG: Electrocardiography, Tpe: T-peak to T-end 
The differences between the maximum and minimum intervals were defined as dispersions (QT dis, QTc dis and Tp-e dis).

As this is a retrospective study, an informed consent was not obtained from the parents' of the participants. Keçiören Training and Research Hospital's scientific committee approved the study and the study was performed in accordance with the principles of Helsinki Declaration (No: 43278876-929, dated: 2020).

\section{Statistical Analysis}

The Statistical package for social sciences program version 21 (SPSS Inc., Chicago, IL, USA) was used for the statistical analyses of the data. Shapiro-Wilk test was used to assess the normal distribution of variables. According to distribution, Student's t-test or Mann-Whitney U test were used to compare groups. The differences in median values between more than two independent groups were analyzed with the Kruskal-Wallis test. A p-value of under 0.05 was considered as the statistical significance level.

\section{Results}

A total of 137 subjects were examined during the study consisting of 56 children with benign ER pattern (Study group) and 81 children with no ER pattern (Control group). There were no differences between the groups in terms of age and gender (Table I). Heart rate was lower in the study group compared to the controls [75.8 (12.3) bpm vs. 81 (15.2) bpm, $p=0.03$ ] (Table II).

In the study group, benign ER was present in inferior derivations in 11 (19.6\%) children, in lateral derivations in $35(62.5 \%)$, and in inferolateral derivations in 10 (17.9\%). QRS slurring was observed in 35 (62.5\%), QRS notching in 18 $(32.1 \%)$ and both in $3(5.4 \%)$ in the study group.

The mean QT and QTc values were found to be higher in the study group, but not at a statistically significant level. The Tp-e interval was significantly higher in the study group [91.3 (11.9) ms, 82.9 (13.6) ms, respectively, p<0.001]. In terms of dispersion of these parameters, all were higher in the study group but only the Tp-e dis value was statistically

Table I. Demographic and clinical characteristics of the study groups

\begin{tabular}{|l|l|l|l|}
\hline & $\begin{array}{l}\text { Study group (with benign ER) } \\
(\mathbf{n = 5 6 )}\end{array}$ & $\begin{array}{l}\text { Control group (without ER) } \\
\text { (n=81) }\end{array}$ & p-value \\
\hline Gender (Male/female) & $48 / 8$ & $59 / 22$ & 0.07 \\
\hline Age (years) & $13.5(3.1)$ & $13.8(2.3)$ & 0.49 \\
\hline Body mass index (kg/m²) & $21(2)$ & $21.3(3.7)$ & 0.77 \\
\hline Systolic blood pressure (mmHg) & $118(6.2)$ & $116.1(6.2)$ & 0.52 \\
\hline Diastolic blood pressure (mmHg) & $71.5(3.9)$ & $67.2(8.7)$ & 0.36 \\
\hline $\begin{array}{l}\text { *Values are presented as mean (SD) } \\
\text { SD: Standard deviation, ER: Early repolarization }\end{array}$ & & & \\
\hline
\end{tabular}

Table II. Comparison of groups according to ECG measurements

\begin{tabular}{|c|c|c|c|}
\hline & $\begin{array}{l}\text { Study group (with benign ER) } \\
(n=56)\end{array}$ & $\begin{array}{l}\text { Control group (without ER) } \\
(n=81)\end{array}$ & p-value \\
\hline Heart rate (bpm) & $75.8(12.3)$ & $81(15.2)$ & 0.03 \\
\hline QT (ms) & $365.3(24)$ & $359(29.1)$ & 0.18 \\
\hline QTc (ms) & $415.7(26.7)$ & $408.9(23)$ & 0.12 \\
\hline Tpe (ms) & $91.3(11.9)$ & $82.9(13.6)$ & $<0.001$ \\
\hline QTdis (ms) & $43.04(14.7)$ & $41.7(14.2)$ & 0.6 \\
\hline QTcdis (ms) & $33.6(18.9)$ & $29.8(17.6)$ & 0.2 \\
\hline Tpedis (ms) & $35.6(15.8)$ & $24.9(12.5)$ & 0.001 \\
\hline Tpe/QT (ms) & $0.25(0.3)$ & $0.23(0.3)$ & 0.03 \\
\hline Tpe/QTc (ms) & $0.22(0.3)$ & $0.20(0.3)$ & $<0.001$ \\
\hline
\end{tabular}


significant [35.6 (15.8) ms in study group, 24.9 (12.5) ms in control group, $\mathrm{p}=0.001]$.

A statistically significant difference was detected between the groups in the ratios of Tp-e/QT and Tp-e/ QTc [0.25 (0.3) ms in study group, $0.23(0.3) \mathrm{ms}$ in control group, $\mathrm{p}=0.03 ; 0.22(0.3) \mathrm{ms}$ in study group, $0.20(0.3) \mathrm{ms}$ in control group, $\mathrm{p}<0.001$ respectively]. The comparisons of the ECG measurements are summarized in Table II.

No significant differences were determined in the studied ECG measurements with respect to ER location (inferior, lateral, inferolateral) and the ER type (slurring, notching or both).

\section{Discussion}

The relationship between malignant arrhythmias and ER has not yet been fully clarified. TDR is one of the related underlying mechanisms. This phenomenon could be described as abnormalities in the ionic mechanism of cardiac cells creating myocardial heterogeneity. During ventricular depolarization, the transient outward potassium current mediated action potential notch is just in the ventricular epicardium and not in the endocardium, which results in a transmural voltage gradient and can be seen as I point elevation on ECG. As a result of this gradient, the phase 2 re-entry of action potential is propagated and/ or adjacent cells activate ER cells for spontaneous activity starting arrhythmogenesis (13).

In previous studies, Tp-e has been reported as a marker of TDR and prolongation of Tp-e has been used to predict arrhythmias, including VT/VF (9). The QT and QTc intervals can also reflect repolarization of total myocardium on ECG. However, Tp-e and Tp-e dis are considered more useful markers of TDR than QT and QTdis (14). The measurements of Tp-e/QT and Tp-e/QTc are also used as a marker of ventricular arrhythmogenesis (10). In addition, these ratios are considered to be more sensitive in the prediction of arrhythmia compared to Tp-e as the constant index of arrhythmogenesis is not affected by heart rate.

In our study, children with benign ER were found to have increased Tp-e, Tp-e dis and Tp-e/QT, Tp-e/QTc ratios compared with the controls. However, there were no statistically significant differences between the groups with respect to QT, QTc and QTdis, QTc dis. In accordance with previous studies, the current study findings also suggest that repolarization changes could be local in children with ER and not affect QT and QTc intervals $(15,16)$. Increased Tp-e, Tp-e dis in different I wave syndromes including Brugada, ER and long QT syndrome have been reported by several adult studies $(16,17)$. However, to the best of our knowledge, these novel indexes of arrhythmogenesis in ER have not been previously investigated in the pediatric age group.

Different studies have pointed out that ER existence depends on autonomic tone and heart rate (16). I point amplitude increases under increased parasympathetic tone and in lower heart rates. In our study, although all the participants were in the same resting condition during ECG, the heart rate was observed to be lower in the study group. Although this result is consistent with the findings of previous studies, the participants were not asked about specific conditions that could affect autonomic tone such as the last time of eating or sleeping time.

The risk of SCD is minimally increased by the ER pattern according to adult studies $(18,19)$. Those adults with an ER pattern in lateral leads, with less than $0.2 \mathrm{mV}$ I wave amplitude, horizontal ST segment and QRS slurring are accepted as having a benign ER pattern and low risk for arrhythmias or SCD (20). In contrast to this absolute definition in adults, the definition and clinical implications of benign ER pattern remain unclear in the pediatric age group, in which the ER pattern is seen 5 to 10 times more often than in the adult population (3).

A previous study with a large sample of children reported that no patient characteristics such as gender, age, body mass index, blood pressure, or previous syncope were found to be markers of ER existence (3). However, there are no data about the risk stratification for arrhythmias and/ or SCD in healthy children with ER. In the present study, no relationship was determined between the ER pattern location, QRS morphology and Tp-e dis, Tp-e/QT, Tp-e/QTc ratios which are known to be predictors of arrhythmia.

\section{Study Limitations}

We are aware of the limitations of this study, primarily the relatively small sample size of the study group. In addition, there was no evaluation of the correlation between ECG measurements and any ventricular arrhythmias (premature ventricular contractions, non-sustained ventricular tachycardia, etc.). Finally, it was not possible to prospectively follow up the study group for future arrhythmic events.

\section{Conclusion}

The findings of our study showed that children with benign ER may have an increased risk of SCD through alterations in ventricular repolarization parameters. This group of patients may be considered at risk for the 
development of arrhythmias. For that reason, there is a need for further prospective and long-term follow-up studies including long term ECG recordings to be able to demonstrate the clinical and prognostic importance of these parameters in children.

\section{Ethics}

Ethics Committee Approval: Keçiören Training and Research Hospital's scientific committee approved the study and the study was performed in accordance with the principles of Helsinki Declaration (No: 43278876-929, dated: 2020).

Informed Consent: As this is a retrospective study, an informed consent was not obtained from the parents' of the participants.

Peer-review: Externally peer-reviewed.

\section{Authorship Contributions}

Concept: S.K., M.K., F.D., Design: S.K., M.K., C.K. Data Collection or Processing: S.K., M.K., C.K., F.D., Analysis or Interpretation: S.K., Literature Search: S.K., Writing: S.K.

Conflict of Interest: No conflict of interest was declared by the authors.

Financial Disclosure: The authors declared that this study received no financial support.

\section{References}

1. Obeyesekere MN, Klein G), Nattel S, et al., A clinical approach to early repolarization. Circulation 2013; 127:1620-9.

2. De Ambroggi L, Sorgente A, De Ambroggi G. Early repolarization pattern: innocent finding or marker of risk? J Electrocardiol 2013; 46:297-301.

3. Ahmed H, Czosek RS, Spar DS, et al. Early repolarization in normal adolescents is common. Pediatr Cardiol 2017; 38:864-72.

4. Benito B, Guasch E, Rivard L, et al. Clinical and mechanistic issues in early repolarization of normal variants and lethal arrhythmia syndromes. J Am Coll Cardiol 2010; 56:1177-86.

5. Haissaguerre $M$, Derval $N$, Sacher $F$, et al. Sudden cardiac arrest associated with early repolarization. N Engl I Med 2008; 358:2016-23.

6. Tikkanen JT, Huikuri HV. Characteristics of "malignant" vs. "benign" electrocardiographic patterns of early repolarization. J Electrocardiol 2015; 48:390-4.
7. Perez-Riera AR, Abreu LC, Yanowitz F, et al. "Benign" early repolarization versus malignant early abnormalities: clinicalelectrocardiographic distinction and genetic basis. Cardiol I 2012; 19:337-46.

8. Merchant FM, Noseworthy PA, Weiner RB, et al. Ability of terminal QRS notching to distinguish benign from malignant electrocardiographic forms of early repolarization. Am J Cardiol 2009; 104:1402-6.

9. Kors JA, Ritsema van Eck HJ, van Herpen $\mathrm{G}$. The m eaning of the Tp-Te interval and its diagnostic value. I Electrocardiol 2008; 41:575-80

10. Gupta P, Patel $\mathrm{C}$, Patel $\mathrm{H}$, et al. T(p-e)/QT ratio as an index of arrhythmogenesis. J Electrocardiol 2008; 41:567-74.

11. Reinhard W, Trenkwalder T, Haller B, et al., The early repolarization pattern: echocardiographic characteristics in elite athletes. Ann Noninvasive Electrocardiol 2019; 24:e12617. doi: 10.1111/anec.12617.

12. Tikkanen JT, Juntilla M), Anttonen O, et al. Early repolarization: electrocardiographic phenotypes associated with favorable long-term outcome. Circulation 2011; 123:2666-73.

13. Antzelevitch C, Yan GX. Cellular and ionic mechanisms responsible for the Brugada syndrome. I Electrocardiol 2000; (Suppl 33):33-9.

14. Karadeniz C, Özdemir R, Demirol M, et al. Low Iron Stores in Otherwise Healthy Children Affect Electrocardiographic Markers of Important Cardiac Events. Pediatr Cardiol 2017; 38:909-14.

15. Tikkanen JT, Anttonen O, Juntilla MJ, et al. Long-term outcome associated with early repolarization on electrocardiography. $\mathrm{N}$ Engl ) Med 2009; 361:2529-37.

16. Letsas KP, Charalampous C, Koantzopoulos P, et al., Novel indexes of heterogeneity of ventricular repolarization in subjects with early repolarization pattern. Europace 2012; 14:877-81.

17. Yamaguchi $M$, Shimizu $M$, Ino $H$, et al. $T$ wave peak-to-end interval and QT dispersion in acquired long QT syndrome: a new index for arrhythmogenicity. Clin Sci (Lond) 2003; 105: 671-6.

18. Maron BJ, Doerer HJ, Haas TS, et al. Sudden deaths in young competitive athletes: analysis of 1866 deaths in the United States, 1980-2006. Circulation 2009; 119:1085-92.

19. Noseworthy PA, Weiner R, Kim J, et al., Early repolarization pattern in competitive athletes: clinical correlates and the effects of exercise training. Circ Arrhythm Electrophysiol 2011; 4:432-40.

20. Mahida S, Sacher F, Berter B, et al. Evaluation of patients with early repolarization syndrome. J Atr Fibrillation 2014; 7:1083. 